\section{Références}

Dourmad J.Y., Étienne M., Noblet J., 1991. Contribution à l'étude des besoins en acides aminés de la truie en lactation. Journées Rech. Porcine en France, 23, 61-68.

Dourmad J.Y., Étienne M., Prunier A., Noblet J., 1994. The effect of energy and protein intake of sows on their longevity : a review. Livest. Prod. Sci., 40, 87-97.

Dourmad J.Y., Étienne M., Noblet J., Causeur D., 1997. Prédiction de la composition chimique des truies reproductrices à partir du poids vif et de l'épaisseur de lard dorsal. Journées Rech. Porcine en France, 29, 255-262.

INRA, 1989. L'alimentation des animaux monogastriques. INRA Editions, Paris.

Noblet J., Dourmad J.Y., Étienne M., 1990. Energy utilization in pregnant and lactating sows : modeling of energy requirements. J. Anim. Sci., 68, 562-572.

Quesnel H., Prunier A., 1995. L'ovulation après le tarissement des truies : mécanismes physiologiques et facteurs de variation. INRA Prod. Anim., 8, 165176.

\title{
Facteurs de variation de l'appétit des truies en lactation
}

Au cours de ces dernières années, le nombre de porcelets produits et sevrés par truie s'est accru. Dans le même temps, l'appétit des truies en lactation n'a pas augmenté, voire a diminué, accentuant ainsi le déficit nutritionnel auquel la femelle est soumise pendant cette période d'exportation intense de nutriments. Ce phénomène pose le problème de l'élaboration d'une stratégie d'alimentation puisqu'il faut faire coïncider les besoins nutritionnels et la capacité des truies à ingérer suffisamment d'aliment. En fait, il apparaît que la consommation moyenne journalière $(\mathrm{CMJ})$ des truies est influencée à la fois par des facteurs intrinsèques de l'animal mais également par des facteurs extrinsèques, liés aux conditions d'élevage. L'étude de ces facteurs a déjà fait l'objet d'une revue par Dourmad (1988), ce texte l'actualise avec les résultats publiés depuis.

Figure 1. Effet marginal de la parité sur la consommation moyenne journalière d'aliment $(C M J)$ pendant la lactation (d'après O'Grady et al 1985).

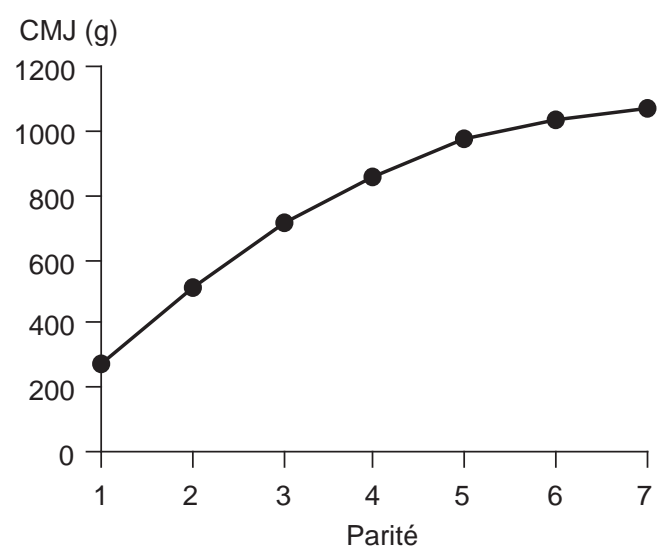

\section{1 / Caractéristiques de la truie}

\section{Numéro de portée}

L'augmentation de la CMJ avec la parité est principalement observée au cours des premières portées. En effet, les travaux de O'Grady et al (1985) mettent en évidence une augmentation curvilinéaire de la CMJ (figure 1) jusqu'à la $6^{\mathrm{e}}$ portée, alors qu'au-delà elle plafonne (Lynch 1989). Cependant, il s'agit de prendre en compte également l'augmentation parallèle de la taille de la portée et du poids de la truie, qui s'accompagnent d'un accroissement des besoins d'entretien et de production et donc potentiellement de la CMJ.

\section{Taille de la portée}

L'augmentation du nombre de porcelets s'accompagne d'un accroissement de la production quotidienne de lait (Elsley 1971) et, souvent, d'une mobilisation accrue des réserves corporelles. En effet, d'après Lynch (1989), la CMJ augmente en moyenne de $200 \mathrm{~g} / \mathrm{j}$ par porcelet alors que le besoin en aliment correspondant serait de 500 à $600 \mathrm{~g}$ (Verstegen et al 1985). L'effet du nombre de porcelets sur la CMJ est cependant de moins en moins important quand la taille de la portée s'accroît (O'Grady et al 1985).

\section{Stade de lactation}

La CMJ augmente avec le stade de lactation pour atteindre un plateau, mais, en pratique, les principales variations de CMJ pendant la lactation sont dues à la conduite alimentaire imposée au cours de la première semaine suivant la mise bas. A partir d'un grand nombre de données collectées sur le terrain, Koketsu et al (1996) ont pu établir six
N. QUINIOU, J.-Y. DOURMAD*, J. NOBLET*

Institut Technique du Porc, La Motte-au-Vicomte, 35650 Le Rheu

: $\square$ INRA, Station de Recherches

Porcines, 35590 Saint-Gilles 
Tableau 1. Répartition des truies ( $n=19$ 393) issues d'élevages du sud du Minnesota et du nord de l'lowa (Etats-Unis) selon la cinétique de prise alimentaire au cours de la lactation (Koketsu et al 1996).

\begin{tabular}{|l|c|}
\hline \multicolumn{1}{|c|}{ Catégorie } & \% dans la population \\
\hline Augmentation rapide après la mise bas & 17,8 \\
Diminution rapide après le pic de consommation & 38,3 \\
Diminution lente après le pic de consommation & 25,8 \\
CMJ faible pendant toute la lactation & 1,2 \\
CMJ faible pendant la première semaine de lactation & 1,5 \\
Augmentation progressive pendant toute la lactation & 15,4 \\
\hline
\end{tabular}

catégories de truies suivant la cinétique de prise alimentaire au cours de la lactation (tableau 1). En fait, d'après Moser et al (1987) et Neil (1996), les truies alimentées à volonté dès la mise bas consomment en moyenne plus d'aliment par jour pendant la lactation que celles rationnées après la mise bas et ce résultat est lié au fait que la quantité d'aliment allouée pendant la première semaine après la mise bas n'influence pas les niveaux de consommation au cours des trois semaines suivantes. La crainte d'agalactie ou de chute d'appétit précoce contribue toutefois, dans les élevages, au maintien d'un rationnement relatif après la mise bas.

\section{Sélection génétique}

De même que pour les porcs en croissance, l'appétit des truies en lactation est influencé par les critères retenus pour la sélection génétique. Ainsi, la sélection génétique pour l'obtention d'animaux de plus en plus maigres n'a pas le même effet sur l'appétit des truies quand elle est réalisée sur la base d'un appétit faible ou sur la base d'un dépôt quotidien de maigre élevé. En effet, après une sélection sur sept générations en condition d'alimentation ad libitum, Kerr et Cameron (1996) constatent que la CMJ pendant la lactation est inférieure de $12 \%$ chez les truies issues d'une lignée sélectionnée pour un faible appétit par rapport aux témoins, alors que les truies en lactation issues d'une lignée sélectionnée pour une vitesse élevée de dépôt de maigre consomment $13 \%$ d'aliment en plus que les truies témoins.

\section{2 / Conditions d'élevage}

La température est la composante climatique dont l'effet est le plus étudié à l'heure actuelle. En effet, les travaux disponibles (Black et al 1993) montrent que la zone de confort thermique de la truie correspond à une plage de température allant de 12 à 20 ${ }^{\circ} \mathrm{C}$, celle du porcelet à la naissance allant de 30 à $35^{\circ} \mathrm{C}$. En pratique, la température ambiante dans les maternités dépasse donc fréquemment la température critique supérieure de la truie. La fréquence des problèmes posés par les températures élevées est souvent liée aux caractéristiques du climat, de la saison, de la période de la journée. Ces pro- blèmes sont encore accentués par la conception des bâtiments et la mauvaise maîtrise de l'ambiance.

\section{Température}

Les résultats disponibles s'accordent sur l'effet négatif des températures élevées sur l'appétit des truies. Le facteur limitant la CMJ semble être alors la capacité de la truie à dissiper sa chaleur interne. Celle-ci peut être réduite via une diminution de la consommation d'aliment et donc de l'extra-chaleur associée (Noblet et al 1985), et par une diminution de la mobilisation des réserves corporelles pour la production laitière (Messias de Bragança et al 1995). La compilation de la bibliographie a permis à Black et al (1993) d'établir qu'une augmentation de $1{ }^{\circ} \mathrm{C}$ de la température ambiante entre 16 et $32 \square^{\circ} \mathrm{C}$ s'accompagne en moyenne d'une diminution de 2,4 MJ d'énergie digestible ingérée par jour. Cependant, la plupart des travaux disponibles comparent deux niveaux constants de température au cours de la lactation. Or, il est probable que l'effet de la température sur la prise alimentaire ne soit pas linéaire. Par ailleurs, aucun résultat ne permet actuellement, à notre connaissance, d'établir si la truie est sensible ou non à des variations nycthémérales de la température selon l'amplitude et la valeur moyenne considérée.

Les systèmes de refroidissement par ventilation spécifique du groin de la truie ou par aspersion d'eau au goutte à goutte sur la tête semblent efficaces pour lutter contre les effets négatifs de la chaleur (McGlone et al 1988).

\section{Lumière}

En comparant des périodes d'éclairage de 8$12 \mathrm{~h}$ et $12-16 \mathrm{~h}$, Prunier et al (1994) constatent que la production laitière des truies est identique pour les deux traitements, mais que l'augmentation de la photopériode est associée à une moindre perte de poids, notamment en période chaude (juin $v s$ janvier). Ce résultat pourrait indiquer un effet favorable de la durée journalière d'éclairement sur la CMJ des truies, y compris lorsque les températures sont élevées, ce qui serait en accord avec un comportement alimentaire essentiellement diurne (Dourmad 1993).

\section{Concentration énergétique et protéique de l'aliment}

Des essais ont été conduits afin de compenser la diminution de CMJ des truies quand la température augmente par un accroissement des teneurs des régimes en nutriments, notamment leur teneur en énergie (Dourmad 1988). Les expériences de Cox et al (1983), Schœnherr et al (1989) et Coffey et al (1994) ont consisté à supplémenter des aliments avec des matières grasses $(+10 \%$ de lipides en moyenne). L'augmentation de la concentration énergétique s'accompagne d'une ingestion d'énergie plus élevée, malgré une CMJ moindre, mais cet apport supplémentaire 
d'énergie correspond presque directement à une exportation plus importante de lipides dans le lait. En définitive, cette stratégie s'accompagne de meilleures performances de croissance de la portée mais n'a pas d'effet probant sur la truie. Une autre voie de recherche permettant de diminuer l'extra-chaleur associée à l'aliment serait de diminuer la teneur en protéines, le besoin en acides aminés essentiels restant couvert en supplémentant l'aliment avec des acides aminés de synthèse. Il s'agit cependant de respecter l'équilibre des apports en acides aminés : un apport trop faible en tryptophane par rapport aux apports de valine et d'isoleucine, par exemple, entraîne une chute de CMJ entre 6 et 12 jours après la mise bas (Trottier et Easter 1995).

\section{Fréquence et présentation des repas}

Libal et Walstrom (1983, cités par Dourmad 1988) ont montré que l'augmentation du nombre de repas de 1 à 3 contribuait à accroître la CMJ des truies de l'ordre de $15 \%$. Par contre, le passage de deux à trois distributions par jour n'a aucun effet sur la CMJ (Genest et D'Allaire 1995). Il est donc possible, compte tenu de ces résultats, que les truies aient deux moments préférentiels de consommation dans la journée. L'humidification des aliments sous forme de farine semble également avoir un effet positif sur la CMJ (+ $12 \%$ : O'Grady et Lynch $1978,+3 \%$ : Danielsen et Nielsen 1984, $5 \%$ : Genest et D'Allaire 1995). Ce système pose cependant des problèmes d'hygiène dus à la fermentation de l'aliment distibué sous forme de soupe, et il reste à vérifier si l'humidification des granulés exerce également un effet positif ou non sur la CMJ.

\section{Alimentation pendant la lactation}

Un niveau d'apport énergétique élevé pendant l'ensemble de la gestation entraîne une diminution de la CMJ pendant la lactation. Ainsi, en accord avec des travaux antérieurs, Dourmad (1993) a montré que l'accroissement du niveau alimentaire de 2,25 à $2,7 \mathrm{~kg} / \mathrm{j}$ pendant la gestation entraîne une chute de la CMJ de $280 \mathrm{~g} / \mathrm{j}$ pendant la lactation, le bilan de gain de poids sur le cycle étant cependant en faveur des truies recevant $2,7 \mathrm{~kg} / \mathrm{j}$ pendant la gestation. La différence de CMJ pendant la lactation doit être mise en relation avec l'état d'engraissement de la truie à la mise bas. A l'inverse, l'accroissement du niveau alimentaire pendant la gestation sans augmentation de l'apport d'énergie (par dilution de l'aliment avec des sources de parois végétales) semble exercer un effet favorable sur la CMJ pendant la lactation (Farmer et al 1996).

\section{Conclusion}

Les facteurs influençant la consommation spontanée des truies en lactation sont nombreux et de natures différentes. De même que le mentionnait Dourmad (1988), il est important de rappeler les difficultés à quantifier les effets respectifs de ces facteurs selon qu'ils sont liés à l'animal, à la conduite d'élevage, à l'aliment... Parmi ces derniers points, l'étude des interactions entre la température et la nature de l'aliment distribué devrait permettre de mieux raisonner les apports de nutriments en fonction de la température et de mieux gérer les répercussions négatives affectant les performances ultérieures de reproduction et la longévité de la truie.

\section{Références bibliographiques}

Black J.L., Mullan B.P., Lorschy M.L., Giles L.R., 1993. Lactation in the sow during heat stress. Livest. Prod. Sci., 35, 153-170.

Coffey M.T., Diggs B.G., Handlin D.L., Knabe D.A. Maxwell C.V.J., Noland P.R., Prince T.J., Gromwell G.L., 1994. Effects of dietary energy during gestation and lactation on reproductive performance of sows ; a cooperative study. J. Anim. Sci., 72, 4-9.

Cox N.M., Britt J.H., Armstrong W.D., Alhusen H.D., 1983. Effect of feeding fat and altering weaning schedule on rebreeding in primiparous sows. J. Anim. Sci., 56, 21-29.

Danielsen V., Nielsen H.E., 1984. The influence of different feeding levels on the performance of lactating sows. In : Proc. 35th annual meeting of the EAAP, 6-9 August 1984, The Hague, The Netherlands, NP3-20.

Dourmad J.-Y., 1988. Ingestion spontanée d'aliment chez la truie en lactation : de nombreux facteurs de variation. INRA Prod. Anim., 1, 141-146.

Dourmad J.-Y., 1993. Standing and feeding behabiour of the lactating sow : effect of feeding level during pregnancy. Appl. Anim. Behav. Sci., 37, 311319 .

Elsley F.W.H., 1971. Nutrition and lactation in the sow. In : I.R. Falconer (ed), Proc. 17th Easter School in Agricultural Science : Lactation, 393-411. Butterworths, London.

Farmer C., Robert S., Matte J.J., 1996. Lactation performance of sows fed a bulky diet during gestation and receiving growth hormone-releasing factor during lactation. J. Anim. Sci., 74, 1298-1306.

Genest M., D’Allaire S., 1995. Feeding strategies during the lactation period for first-parity sows. Can. J. Anim. Sci., 75, 461-467.

Kerr J.C., Cameron N.D., 1996. Responses of gilt post-farrowing traits and pre-weaning piglet growth to divergent selection for components of efficient lean growth rate. Anim. Sci., 63, 523-531.

Koketsu Y., Dial G.D., Pettigrew J.E., Marsch W.E. King V.L., 1996. Influence of imposed feed intake patterns during lactation on reproductive performance and on circulating levels of glucose, insulin and lutheinizing hormone in primiparous sows. J. Anim. Sci., 74, 1036-1046. 
Lynch P.B., 1989. Voluntary food intake of sows and gilts. In : A.T. Smith and T.L.J. Lawrence (eds), Pig housing and the environment, 71-77. Brit. Soc. Anim. Prod., Edinburgh.

McGlone J.J., Stansbury W.F., Tribble L.F., 1988. Management of the lactating sow during heat stress : effects of water dript, snout coolers, floor type and a high energy-density diet. J. Anim. Sci., 66, 885-891.

Messias de Bragança M., Quesnel H., Mounier A.-M., Prunier A., 1995. Influence de la température ambiante sur les performances zootechniques et certains paramètres sanguins chez des truies Large White primipares. Journées Rech. Porcine en France, 27, 37-44.

Moser R.L., Cornelius S.G., Pettigrew J.E., Hanke H.E., Heeg T.R., Miller K.P., 1987. Influence of postpartum feeding method on performance of the lactating sow. Livest. Prod. Sci., 16, 91-99.

Neil M., 1996. Ad libitum lactation feeding of sows introduced immediately before, at, or after farrowing. Anim. Sci., 63, 497-505.

Noblet J., Le Dividich J., Bikawa T., 1985. Interaction between energy level in the diet and environmental temperature on the utilization of energy in growing pigs. J. Anim. Sci., 61, 452-459.
O'Grady J.F., Lynch P.B., 1978. Voluntary feed intake by lactating sows : influence of system of feeding an nutrient density of the diet. Ir. J. Agric. Res., $17,1-5$

O’Grady J.F., Lynch P.B., Kearney P.A., 1985. Voluntary feed intake by lactating sows. Livest. Prod. Sci. $12,355-365$.

Prunier A., Dourmad J.-Y., Etienne M., 1994. Effect of light regimen under various ambiant temperatures on sow and litter performance. J. Anim. Sci., $72,1461-1466$

Schœnherr W.D., Stahy T.S., Cromwell G.L., 1989 The effects of dietary fat or fiber addition on yield and composition of milk from sows housed in warm or hot environment. J. Anim. Sci., 67, 482-495.

Trottier N.L., Easter R.A., 1995. Dietary and plasma branched-chain amino acids in relation to tryptophan : effect on voluntary feed intake and lactation metabolism in the primiparous sow. J. Anim. Sci. 73, 1086-1092.

Verstegen M.W.A., Mesu J., van Kempen G.J.M., Geerse C., 1985. Energy balances of lactating sows in relation to feeding level and stage of lactation. $\mathrm{J}$. Anim. Sci., 60, 731-740.

\section{ETIENNE, \\ M.-C. PERE}

INRA Station

de Recherches

Porcines, 35590 Saint-Gilles

\section{Adaptations physiologiques et métaboliques au cours de la gestation chez la truie}

\section{Apports nutritionnels et performances de reproduction}

Les quantités d'énergie ou de protéines allouées aux truies gravides n'affectent pas en général la taille de la portée. En revanche, l'augmentation des apports énergétiques accroît le poids du porcelet à la naissance (Henry et Etienne 1978). L'effet est plus marqué chez les truies multipares $(+37 \mathrm{~g} / \mathrm{Mcal} ; \mathrm{R}$ $=0,65)$ que chez les primipares $(+15 \mathrm{~g} /$ Mcal ; $\mathrm{R}=0,30)$. Seules des conditions extrêmes d'apports de protéines (aliments composés quasi exclusivement de céréales régimes protéiprives) provoquent une réduction de poids des porcelets nouveau-nés (Duée et Sève 1978).

\section{Adaptations maternelles et fœtales}

\section{Capacité utérine}

La truie n'est capable de conduire à terme qu'un nombre limité de fotus. Cette limitation, appelée capacité utérine, est caractéris- tique du type génétique et de l'individu. Différents modèles permettant de faire varier le nombre potentiel d'embryons ont été utilisés. La régularisation de la taille de la portée intervient précocement, généralement avant 35 jours de gestation. Cependant, lorsque l'effectif potentiel (taux d'ovulation) est important, des différences persistent à ce stade, et la mortalité tardive des fotus est accrue. Les effets d'une augmentation ou d'une restriction de l'espace utérin disponible par fotus sur le développement de la portée ont été étudiés en comparant des truies témoins (CTR), des femelles HHO ayant subi une hémi-hystéroovariectomie droite (l'effectif initial d'embryons dans la corne utérine subsistante est deux fois plus important que dans chacune des cornes des truies du lot CTR), et des truies LIG dont l'oviducte gauche était ligaturé (l'effectif initial d'embryons dans chacune des deux cornes utérines est deux fois plus faible que celui du lot CTR). La figure 1 présente l'évolution du nombre de fœtus par corne utérine pendant la gestation dans les trois groupes expérimentaux. A 35 jours de gestation, la hiérarchie du nombre de fotus par corne utérine est la même qu'au début, mais les écarts entre les groupes sont fortement atténués en raison d'une mortalité 